\title{
Seismic Code Analysis of Buildings without Locating Centers of Rigidity
}

\author{
By Rakesh K. Goel, ' and Anil K. Chopra ${ }^{2}$
}

\begin{abstract}
Static force procedures of most building codes require that the lateral earthquake force at each floor level of an asymmetric-plan building be applied eccentrically from the centers of rigidity. To implement such procedures, it seemed necessary in the past to determine the locations of the centers of rigidity, an often confusing and cumbersome process. This paper presents a new approach for code lateral force analysis of asymmetric-plan buildings without locating the centers of rigidity. This procedure combines the results from three static analyses, which can be implemented directly on most commercially available computer programs for analysis of multistory buidings. The work presented in this paper should dispel the long-held view that locations of the centers of rigidity must be determined to implement the code procedure, thereby removing one of the major difficulties in building analysis.
\end{abstract}

\section{INTRODUCTION}

Static force procedures of most building codes require that the lateral earthquake force at each floor level of an asymmetric-plan building be applied eccentrically from the center of rigidity at a distance equal to the design eccentricity (Earthquake 1992). The design eccentricity $e_{d j}$ to be used at the $j$ th floor level is specified in most seismic codes as

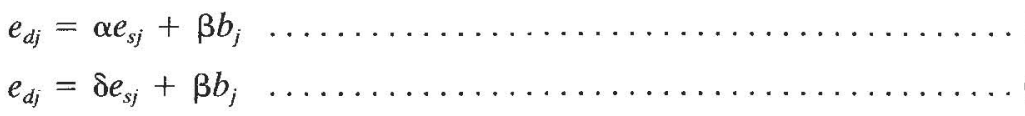

where $e_{s j}=$ eccentricity for the $j$ th floor defined as the distance between the floor center of mass (CM) and center of rigidity (CR); $b_{j}=$ floor-plan dimension of the building perpendicular to the direction of ground motion; and $\alpha, \beta$, and $\delta=$ specified coefficients. For each structural frame or wall the $e_{d j}$ value leading to the larger design force is to be used. The first term in (1) involving $e_{s j}$ is intended to account for the coupled lateral-torsional response of the building arising from lack of symmetry in plan. The second term, which is often called the accidental eccentricity and is specified as a fraction of the plan dimension $b_{j}$, is included to consider torsional effects due to other factors, such as the rotational component of ground motion about a vertical axis; differences between computed and actual values of stiffnesses, yield strengths, and dead-load masses; and unforeseeable unfavorable distribution of live-load masses.

Several codes, such as Uniform Building Code (1991), "Tentative Provisions for the Development of Seismic Regulations for Buildings" (1978), and New Zealand Standard NZS 4230 (1984), specify that the lateral force

${ }^{1}$ Visiting Asst. Res. Engrg., Dept. of Civ. Engrg., Univ. of California, Berkeley, CA 94720.

${ }^{2}$ Prof., Dept. of Civ. Engrg., Univ. of California, Berkeley, CA. 


\title{
Seismic Code Analysis of Buildings without Locating Centers of Rigidity
}

\author{
By Rakesh K. Goel, ${ }^{1}$
}

and Anil K. Chopra ${ }^{2}$

\begin{abstract}
Static force procedures of most building codes require that the lateral earthquake force at each floor level of an asymmetric-plan building be applied eccentrically from the centers of rigidity. To implement such procedures, it seemed necessary in the past to determine the locations of the centers of rigidity, an often confusing and cumbersome process. This paper presents a new approach for code lateral force analysis of asymmetric-plan buildings without locating the centers of rigidity. This procedure combines the results from three static analyses, which can be implemented directly on most commercially available computer programs for analysis of multistory buidings. The work presented in this paper should dispel the long-held view that locations of the centers of rigidity must be determined to implement the code procedure, thereby removing one of the major difficulties in building analysis.
\end{abstract}

\section{INTRODUCTION}

Static force procedures of most building codes require that the lateral earthquake force at each floor level of an asymmetric-plan building be applied eccentrically from the center of rigidity at a distance equal to the design eccentricity (Earthquake 1992). The design eccentricity $e_{d j}$ to be used at the $j$ th floor level is specified in most seismic codes as

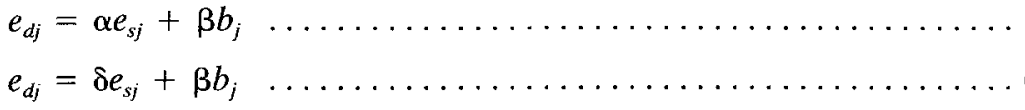

where $e_{s j}=$ eccentricity for the $j$ th floor defined as the distance between the floor center of mass (CM) and center of rigidity (CR); $b_{j}=$ floor-plan dimension of the building perpendicular to the direction of ground motion; and $\alpha, \beta$, and $\delta=$ specified coefficients. For each structural frame or wall the $e_{d j}$ value leading to the larger design force is to be used. The first term in (1) involving $e_{s j}$ is intended to account for the coupled lateral-torsional response of the building arising from lack of symmetry in plan. The second term, which is often called the accidental eccentricity and is specified as a fraction of the plan dimension $b_{j}$, is included to consider torsional effects due to other factors, such as the rotational component of ground motion about a vertical axis; differences between computed and actual values of stiffnesses, yield strengths, and dead-load masses; and unforeseeable unfavorable distribution of live-load masses.

Several codes, such as Uniform Building Code (1991), "Tentative Provisions for the Development of Seismic Regulations for Buildings" (1978), and New Zealand Standard NZS 4230 (1984), specify that the lateral force

${ }^{1}$ Visiting Asst. Res. Engrg., Dept. of Civ. Engrg., Univ. of California, Berkeley, CA 94720.

${ }^{2}$ Prof., Dept. of Civ. Engrg., Univ. of California, Berkeley, CA. 
be applied at a distance equal to $\pm \beta b_{j}$ from the $\mathrm{CM}$, which is equivalent to $\alpha=\delta=1$ in (1). In implementing the lateral-force provisions of such codes, it is not necessary to determine the locations of the CRs at the various floor levels. However, for codes in which the design eccentricity formula implies $\alpha$ or $\delta$ different than 1, e.g., National Building Code of Canada (1990) and the 1987 Mexico Federal District Code (Gomez and Garcia-Ranz 1988), it seems necessary to determine the locations of the CRs. This has been the long-held view that has motivated much work on clarification of the CR concept and procedures for locating the CRs for multistory buildings (Cheung and Tso 1986; Hejal and Chopra 1987; Humar 1984; Pool 1977; Riddell and Vasquez 1984; Tso 1990).

Unlike one-story buildings, there are several difficulties in establishing locations of the CR at various floor levels of a multistory building unless it belongs to a special class known as proportional buildings (Cheung and Tso 1986; Hejal and Chopra 1987; Riddell and Vasquez 1984). First, there is no generally accepted definition of the CRs. Poole (1977) defined the CR of a story as the location of the resultant of shear forces of various resisting elements in that story when the building is subjected to a static lateral loading that causes no rotation in any of the stories. Humar (1984) interpreted the $\mathrm{CR}$ at any floor as a point such that application of a lateral load through this point would not cause rotation of that floor; other floors may rotate. However, Cheung and Tso (1986) defined the CRs as the set of points located on the building floors through which application of lateral forces would cause no rotation of any of the floors. Second, for a chosen definition of the CRs, their location depends on the heightwise distribution of lateral forces. Third, it is cumbersome to determine the CRs for a complex multistory building. Most commercially available computer programs for structural analysis do not have the capabilities of the matrix calculations required to determine the CRs by available procedures (Cheung and Tso 1986; Hejal and Chopra 1987). Therefore, simplified methods have been developed for computing the CRs from the results of plane-frame analysis programs (Poole 1977; Tso 1990) by supplemental analysis requiring hand calculation or special-purpose postprocessing computer programs.

Given all these difficulties in locating the CRs, it is cumbersome to implement the code static procedure for many asymmetric-plan, multistory buildings. Therefore, it is desirable to develop an analysis approach that avoids explicit determination of the CRs. This paper is aimed towards filling this need. Presented is a new approach that eliminates the need for explicit computation of the CRs and yet leads to results identical to those obtained by the approach in which CRs are computed explicitly. The results from static analyses for three sets of forces applied at the CMs are combined appropriately to determine the design forces. The three static analyses can be directly carried out on most commercially available computer analysis programs.

In this paper, the third of the three aforementioned definitions for the CRs (Cheung and Tso 1986) is adopted. The procedure to implement the static procedure using these CRs is reviewed first to demonstrate the difficulties in implementing such an approach followed by description of the new approach. Various steps involved in the two approaches are illustrated with a numerical example. The equivalence of the two approaches is demonstrated conceptually by the principle of superposition; a more formal mathematical proof is presented in Appendix I. 


\section{ANALYSIS USING CENTERS OF RIGIDITY}

The first step in implementing this approach is to determine the locations of the CRs for all floor levels of the multistory building by either the matrix approach (Cheung and Tso 1986; Hejal and Chopra 1987) or the plane frame analysis (Poole 1977; Tso 1990).

\section{Computation of Centers of Rigidity}

\section{Matrix Approach}

Consider an $N$-story building with orthogonal arrangement of lateral-load resisting elements connected by rigid floor diaphragms (Fig. 1). For lateral force analysis in the $y$-direction, the building plan is treated as symmetric

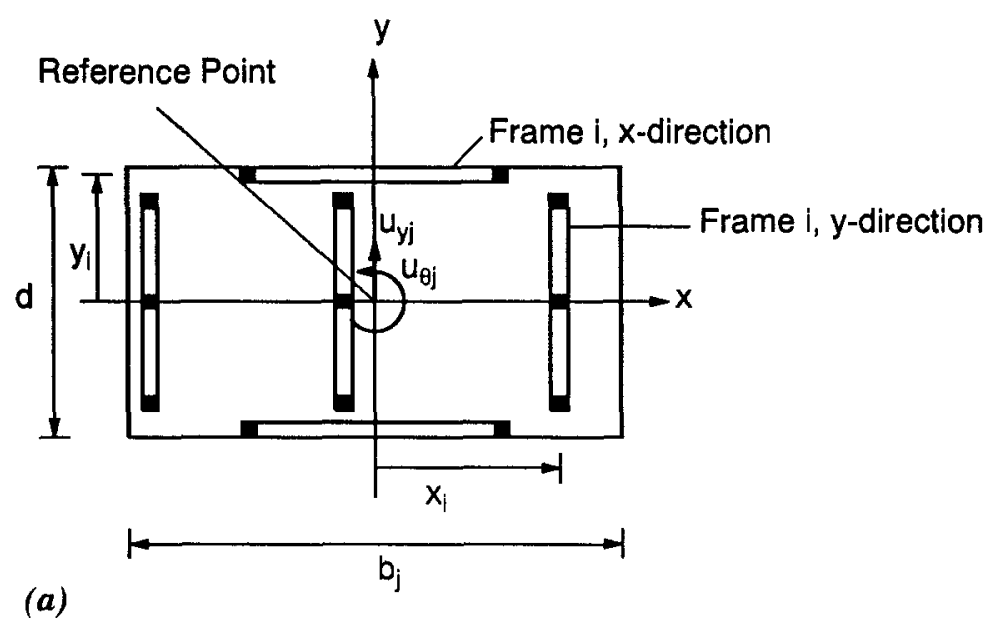

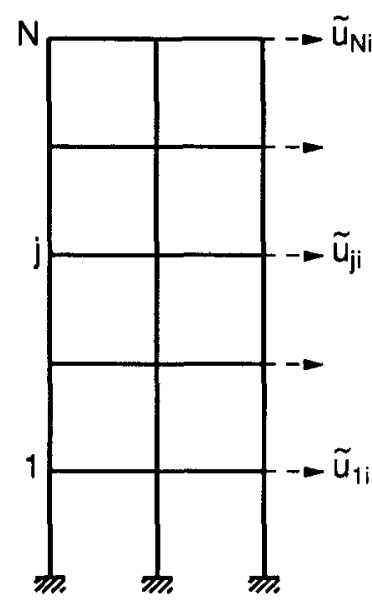

(b) ith frame in $y$-direction

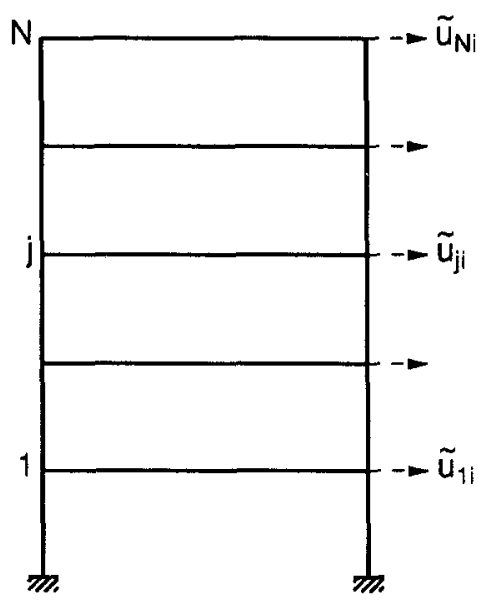

(c) ith frame in $x$-direction

FIG. 1. Multistory System Considered 
about the $x$-axis without the loss of generality because building codes require such independent analyses in the $x$ - and $y$-directions. The equilibrium equations for such systems are

$$
\left[\begin{array}{ll}
\mathbf{K}_{y y} & \mathbf{K}_{y \theta} \\
\mathbf{K}_{\theta y} & \mathbf{K}_{\theta \theta}
\end{array}\right]\left\{\begin{array}{l}
\mathbf{u}_{y} \\
\mathbf{u}_{\theta}
\end{array}\right\}=\left\{\begin{array}{l}
\mathbf{F}_{y} \\
\mathbf{F}_{\theta}
\end{array}\right\}
$$

in which the displacement vectors $=\mathbf{u}_{y}^{r}=\left\langle u_{y 1}, u_{u 2}, \ldots, u_{y j}, \ldots, u_{y N}\right\rangle$ and $\mathbf{u}_{\theta}^{T}=\left\langle u_{\theta 1}, u_{\theta 2}, \ldots, u_{\theta j}, \ldots, u_{\theta N}\right\rangle$, where $u_{y j}=y$-lateral displacement and $u_{8 j}=$ torsional displacement at a selected reference point on the $j$ th floor; and the vectors of floor forces are $\mathrm{F}_{y}^{T}=\left\langle F_{y 1}, F_{y 2}, \ldots, F_{y i}, \ldots\right.$, $\left.F_{y N}\right\rangle$, and $\mathbf{F}_{\theta}^{T}=\left\langle F_{\theta 1}, F_{\theta 2}, \ldots, F_{\theta j}, \ldots, F_{\theta N}\right\rangle$ where $\mathbf{F}_{y j}=$ lateral force in $y$-direction and $\mathbf{F}_{\theta j}=$ floor torque applied at the selected reference point on the $j$ th floor.

Various submatrices of the stiffness matrix in (2) can be expressed in terms of the lateral stiffness matrices of the individual resisting elements (frames or shear walls) as follows:

$$
\begin{aligned}
& \mathbf{K}_{y y}=\sum_{i} \mathbf{k}_{y i} \ldots \ldots \ldots \\
& \mathbf{K}_{y \theta}=\mathbf{K}_{\theta y}=\sum_{i} x_{i} \mathbf{k}_{y i} \cdots \\
& \mathbf{K}_{\theta \theta}=\sum_{i} x_{i}^{2} \mathbf{k}_{y i}+\sum_{i} y_{i}^{2} \mathbf{k}_{x i}
\end{aligned}
$$

where $x_{i}=$ distance from the reference point to the $i$ th frame oriented in the $y$-direction with lateral stiffness matrix $\mathbf{k}_{y i}$; and $y_{i}=$ distance from the reference point to the $i$ th frame oriented in the $x$-direction with lateral stiffness matrix $\mathbf{k}_{x i}$.

To determine the locations of the CRs, we rewrite the equilibrium equations with respect to the degrees of freedom $\tilde{\mathbf{u}}^{T}=\left\langle\tilde{\mathbf{u}}_{y}^{T} \tilde{\mathbf{u}}_{\theta}^{T}\right\rangle$ at the CRs as

$$
\left[\begin{array}{ll}
\mathbf{K}_{y y} & \left.\mathbf{K}_{y \theta}-\mathbf{K}_{y y} \mathbf{X}_{R}\right) \\
\left(\mathbf{K}_{\theta y}-\mathbf{X}_{R} \mathbf{K}_{y y}\right) & \left(\mathbf{K}_{\theta \theta}+\mathbf{X}_{R} \mathbf{K}_{y y} \mathbf{X}_{R}-\mathbf{X}_{R} \mathbf{K}_{y \theta}-\mathbf{K}_{\theta y} \mathbf{X}_{R}\right)
\end{array}\right]\left\{\begin{array}{l}
\tilde{\mathbf{u}}_{y} \\
\tilde{\mathbf{u}}_{\theta}
\end{array}\right\}=\left\{\begin{array}{l}
\mathbf{F}_{y} \\
\tilde{\mathbf{F}}_{\theta}
\end{array}\right\}
$$

in which $\mathbf{X}_{R}=$ diagonal matrix with its diagonal elements $=x_{R j}$, where $x_{R j}$ $=x$-coordinate of the $C R$ of the $j$ th floor; and $\overline{\mathbf{F}}_{\theta}=\mathbf{F}_{\theta}-\mathbf{X}_{R} \overrightarrow{\mathbf{F}}_{y}=$ vector of floor torques at the CR.

From the definition of the centers of rigidity (Cheung and Tso 1986; Hejal and Chopra 1987), if only lateral forces are applied at the CRs, i.e. $\hat{\mathbf{F}}_{\theta}=$ 0 , the system would undergo pure translation, i.e. $\tilde{\mathbf{u}}_{\theta}=0$. Thus, (4) becomes

$\mathbf{K}_{y y} \overline{\mathbf{u}}_{y}=\mathbf{F}_{y}$

$\left(\mathbf{K}_{\theta y}-\mathbf{X}_{R} \mathbf{K}_{y y}\right) \tilde{\mathbf{u}}_{y}=0$

Eq. (5a) is solved for $\overline{\mathbf{u}}_{y}$

$$
\tilde{\mathbf{u}}_{y}=\mathbf{K}_{y y}^{-1} \mathbf{F}_{y}
$$

and $(6)$ is substituted in $(5 b)$ to obtain

$$
\left(\mathbf{K}_{\theta y}-\mathbf{X}_{R} \mathbf{K}_{y y}\right)\left(\mathbf{K}_{y y}^{-1}\right) \mathbf{F}_{y}=0
$$

For the special class of buildings mentioned previously, the locations of 
the CRs are independent of the lateral force distribution. Therefore, (7) implies that

$\left(\mathbf{K}_{\theta y}-\mathbf{X}_{R} \mathbf{K}_{y y}\right)\left(\mathbf{K}_{y y}^{-1}\right)=0$

from which the diagonal matrix $\mathbf{X}_{R}$ defining the locations of the CRs can be detemined

$\mathbf{X}_{R}=\mathbf{K}_{\theta y} \mathbf{K}_{y y}^{-1}$

For buildings not belonging to the special class, the location of the CR may depend on the lateral force distribution, in which case (7) may be written as

$\mathbf{K}_{\theta y} \mathbf{K}_{y y}^{-1} \mathbf{F}_{y}=\mathbf{X}_{R} \mathbf{F}_{y}$

which can be solved to determine

$\mathbf{x}_{R}=\left[\mathbf{F}_{y}\right]^{-1} \mathbf{K}_{\theta y} \mathbf{K}_{y y}^{-1} \mathbf{F}_{y}$

where $\mathbf{x}_{R}=$ vector of $x_{R j} ;\left[\mathbf{F}_{y}\right]=$ diagonal matrix of $F_{y j}$; and $\mathbf{F}_{y}=$ vector of $F_{y j}$. The vector $\mathbf{F}_{y}$ used in (11) is usually taken as the vector of lateral forces specified by the building code.

To illustrate the procedure for computing the floor centers of rigidity, consider a four-story building having three frames A, B, and C spanning in the $y$-direction connected through rigid floor diaphragms (Fig. 2); the building is symmetric in the $x$-direction. This example building possesses stiffness only in the $y$-direction. Most actual buildings, however, would also possess stiffness in the $x$-direction; for such buildings, this stiffness would have to be considered in $(3 c)$. All beams in the frames are identical, with moment of inertia $I_{b}=0.3 \mathrm{~m}^{4}$. The column moment of inertia is $0.1 \mathrm{~m}^{4}$ for frame $B$ and $0.05 \mathrm{~m}^{4}$ for frames A and C. The columns are assumed to be axially rigid. The floor weights are $20 \mathrm{kN}$ for each of the bottom two floors and $10 \mathrm{kN}$ for the top two floors. This example building is the same as that used by Tso (1990).

This example building has large discontinuity in stiffness and mass between the second and third stories. Consequently, it may not satisfy the code criteria for the equivalent static procedure to be applicable. This build-

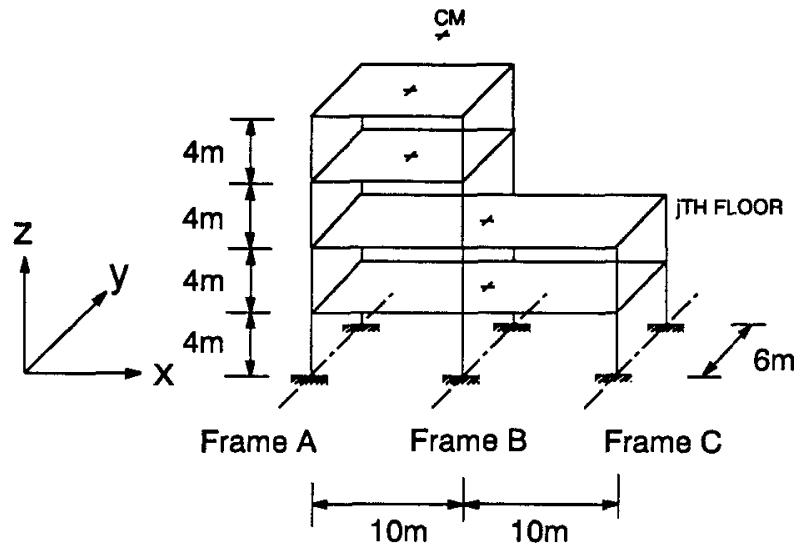

FIG. 2. Example Building 
ing is chosen here, however, for the purpose of illustrating two analysis approaches and their equivalence, and should not be considered as an example where the static code procedure is necessarily applicable.

The example building is designed as per the seismic provisions of the 1987 Mexico Federal District Code (Gomez and Garcia-Ranz 1988). For the seismic coefficient $c=0.6$ and yield reduction factor $Q=2$, the base shear $V_{b}$ for this example building is $=(0.6)(60) /(2)=18 \mathrm{kN}$. The lateral forces $\mathbf{F}_{y}$ are computed as illustrated in Table 1.

The lateral stiffness matrices of frames $\mathrm{A}, \mathrm{B}$, and $\mathrm{C}$ are determined by structural analysis program

$$
\begin{aligned}
& \mathbf{k}_{y A}=\frac{E}{100}\left[\begin{array}{rrrr}
3.57 & -1.87 & 0.18 & -0.01 \\
-1.87 & 3.40 & -1.86 & 0.16 \\
0.18 & -1.86 & 3.37 & -1.67 \\
-0.01 & 0.16 & -1.67 & 1.52
\end{array}\right] \\
& \mathbf{k}_{y B}=\frac{E}{100}\left[\begin{array}{rrrr}
6.93 & -3.75 & 0.57 & -0.05 \\
-3.75 & 6.37 & -3.68 & 0.50 \\
0.57 & -3.68 & 6.23 & -3.06 \\
-0.05 & 0.50 & -3.06 & 2.61
\end{array}\right] \\
& \mathbf{k}_{y C}=\frac{E}{100}\left[\begin{array}{rrrr}
3.55 & -1.69 & 0 & 0 \\
-1.69 & 1.53 & 0 & 0 \\
0 & 0 & 0 & 0 \\
0 & 0 & 0 & 0
\end{array}\right] \ldots \ldots
\end{aligned}
$$

Substituting (12)-(14) in (3a) and (3b) with location of the frame $\mathrm{A}$ as the reference point give

$$
\begin{aligned}
\mathbf{K}_{y y} & =\frac{E}{100}\left[\begin{array}{rrrr}
14.05 & -7.31 & 0.74 & -0.06 \\
-7.31 & 11.29 & -5.54 & 0.66 \\
0.74 & -5.54 & 9.60 & -4.73 \\
-0.06 & 0.66 & -4.73 & 4.13
\end{array}\right] \ldots \\
\mathbf{K}_{y \theta} & =\frac{E}{100}\left[\begin{array}{rrrr}
140.22 & -71.21 & 5.67 & -0.51 \\
-71.21 & 94.12 & -36.79 & 4.98 \\
5.67 & -36.79 & 62.28 & -30.59 \\
-0.51 & 4.98 & -30.59 & 26.06
\end{array}\right]
\end{aligned}
$$

\begin{tabular}{|c|c|c|c|c|}
\hline $\begin{array}{l}\text { Floor } \\
j \\
(1)\end{array}$ & $\begin{array}{l}\text { Height } \\
h_{j} \\
(2)\end{array}$ & $\begin{array}{c}\text { Weight } \\
w_{j} \\
(3)\end{array}$ & $\begin{array}{c}w_{j} h_{j} \\
\text { (4) }\end{array}$ & $F_{y j}=\frac{w_{j} h_{j}}{\sum_{(5)} w_{j} h_{j}} V_{b}$ \\
\hline 4 & 16 & 10 & 160 & 5.54 \\
\hline 3 & 12 & 10 & 120 & 4.15 \\
\hline 2 & 8 & 20 & 160 & 5.54 \\
\hline 1 & 4 & 20 & 80 & 2.77 \\
\hline
\end{tabular}

Using these matrices along with the lateral force vector $\mathbf{F}_{y}^{T}=\langle 2.77,5.54$, $4.15,5.54\rangle$ in (11), the locations of the floor CRs are given as

TABLE 1. Heightwise Distribution of Lateral Forces 
$\mathbf{x}_{R}=\left\{\begin{array}{r}8.55 \\ 17.57 \\ 6.14 \\ 5.99\end{array}\right\}$

This matrix approach to locate the CRs is not convenient in practice, because most standard analysis computer programs do not have the capabilities to implement the matrix manipulation required in (9) and (11). It is necessary, therefore, to develop special programs to implement these calculations.

\section{Plane Frame Analysis Approach}

The location of the CR at each floor can also be determined from equilibrium analysis of the free-body diagram of that floor when the lateral forces $\mathbf{F}_{y}$ are applied at the floor levels in such a manner that no rotation of any of the floors occurs (Tso 1990). To illustrate this process, free-body diagrams of various floors of the example building are shown in Fig. 3. Let $V_{i A}, V_{j B}$, and $V_{j C}(j=1,2,3,4)$ be the $j$ th story shears in frames $\mathrm{A}, \mathrm{B}$, and $C$, respectively. For buildings with rigid diaphragm and with orthogonal arrangement of lateral load resisting elements (frames, walls, and so forth), $V_{j A}, V_{j B}$, and $V_{j C}$ can be computed by analysis of a plane-frame model of the building to the given set of lateral forces. The plane-frame model of the building (Fig. 4) is obtained by arranging all frames (or shear walls) spanning in the same direction side-by-side and linking them at all floor levels by hinge-ended rigid links to simulate rigid floor diaphragm action without rotation (Poole 1977; Tso 1990). Equilibrium of forces in the $y$-direction at each floor level leads to

$$
\left(V_{j A}-V_{j+1, A}\right)+\left(V_{j B}-V_{j+1, B}\right)+\left(V_{j C}-V_{j+1, C}\right)=\mathbf{F}_{y j}
$$

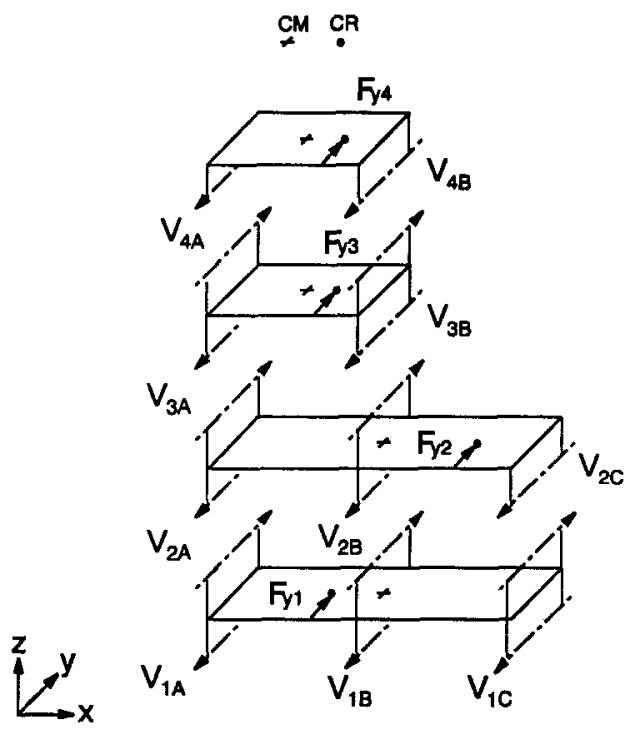

FIG. 3. Free-Body Diagram of Each Floor [After Tso (1990)] 


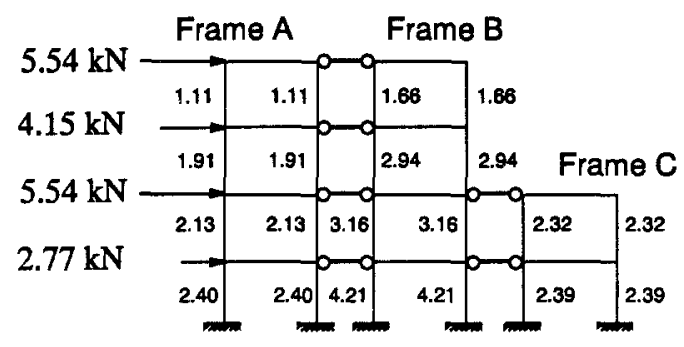

FIG. 4. Plane-Frame Model of Example Building

TABLE 2. Story Shears in Various Frames of Example Building

\begin{tabular}{c|c|c|c}
\hline \hline $\begin{array}{c}\text { Floor } j \\
(1)\end{array}$ & $\begin{array}{c}\text { Frame A } \\
(\mathrm{kN}) \\
(2)\end{array}$ & $\begin{array}{c}\text { Frame B } \\
(\mathrm{kN}) \\
(3)\end{array}$ & $\begin{array}{c}\text { Frame C } \\
(\mathrm{kN}) \\
(4)\end{array}$ \\
\hline 4 & 2.22 & 3.32 & - \\
3 & 3.82 & 5.88 & - \\
2 & 4.26 & 6.32 & 4.64 \\
1 & 4.80 & 8.42 & 4.78 \\
\hline
\end{tabular}

where $\mathbf{F}_{y j}=$ the lateral force at the $j$ th floor. Equilibrium of moments about a vertical axis gives the location of the CR at floor level $j$ as

$x_{R j}=\frac{\left(V_{j A}-V_{j+1, A}\right) x_{A}+\left(V_{j B}-V_{j+1, B}\right) x_{B}+\left(V_{j C}-V_{j+1, C}\right) x_{C}}{F_{y j}} \ldots$

in which $x_{A}, x_{B}$, and $x_{C}=x$-distances of frames $\mathrm{A}, \mathrm{B}$, and C, respectively, from the reference point.

For the example building, shears in columns of frames A, B and C in the plane-frame model due to the lateral forces $\mathbf{F}_{y}^{T}=\langle 2.77,5.54,4.15,5.54\rangle$, obtained from a standard computer program (Wilson and Habibullah 1984) are shown on Fig. 4. The shear in a particular story of a frame is obtained by summing the column shears in the frame (Table 2). For example, the shear in second story of frame $A=2.13+2.13=4.26 \mathrm{kN}$. Shears in other stories of frame A as well as in frames B and C are summarized in Table 2. Locations of the CRs, as measured from frame $\mathrm{A}$, can then be computed from (19). For example, the location of the CR at the second floor is given by:

$x_{R 2}=\frac{(4.26-3.82)(0)+(6.32-5.88)(10)+(4.64)(20)}{5.54}=17.55 \mathrm{~m}$

and the eccentricity of the CR at the second floor relative to the CM is given by

$e_{R 2}=17.55-10=7.55 \mathrm{~m}$

The locations of the floor CRs and floor eccentricities for all floors are summarized in Table 3.

The analysis to determine the shears in the columns of various frames can be carried out on many computer programs for structural analysis. Most 
TABLE 3. Location of Centers of Rigidity and Floor Eccentricities for Example Building

\begin{tabular}{c|c|c}
\hline \hline $\begin{array}{c}\text { Floor } \\
j\end{array}$ & $\begin{array}{c}\text { Location of center of rigidity } \\
(\mathrm{m}) \\
(1)\end{array}$ & $\begin{array}{c}\text { Floor eccentricity } \\
(\mathrm{m}) \\
(3)\end{array}$ \\
\hline 4 & 5.99 & 0.99 \\
3 & 6.17 & 1.17 \\
2 & 17.55 & 7.55 \\
1 & 8.59 & -1.41 \\
\hline
\end{tabular}

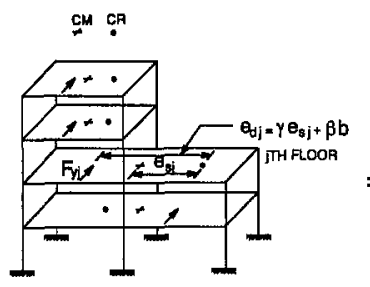

(a)

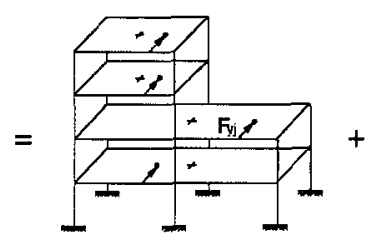

(b)

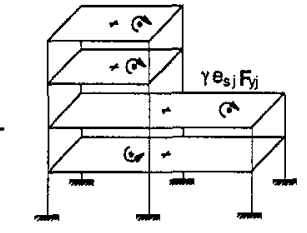

(c)

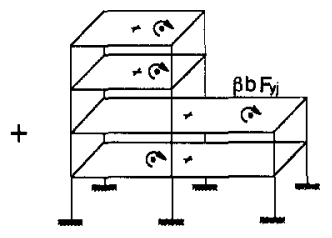

(d)

FIG. 5. Analysis Using Centers of Rigidity

of these programs, however, do not have the capabilities to carry out the additional computations (described in this section) to arrive at the locations of the CRs. Special-purpose postprocessing computer programs are necessary to implement this specific task.

\section{Implementation of Code Static Procedure}

Having determined the locations of the CRs at various floor levels, the code static procedure can be implemented by applying the lateral forces $F_{y j}$ at a distance equal to $e_{d j}$ from the CR at each floor [Fig. 5(a)]. This implies the need for two analyses: $e_{d j}$ is defined by $(1 a)$ for the first analysis and by $(1 b)$ for the second analysis. These two definitions can be written in a condensed form as

$e_{d j}=\gamma e_{s j} \pm \beta b_{j}$

in which $\gamma=\alpha$ and $+\beta b_{j}$ to arrive at $(1 a)$; and $\gamma=\delta$ and $-\beta b_{j}$ to arrive at $(1 b)$. For buildings with rigid diaphragms, the load condition of Fig. $5(a)$ is equivalent to superposition of three load cases: (1) Lateral forces $F_{y j},(j$ $=1,2, \ldots, N)$ at the floor CRs [Fig. $5(b)] ;(2)$ floor torques $=\gamma e_{s j} F_{y j}$ [Fig. 5(c)]; and (3) floor torques $=\beta b_{j} F_{y j}[$ Fig. $5(d)]$. While conceptually useful to separate floor torques into two parts for the purpose of structural 
analysis, the floor torques of the second and third load cases need not be considered separately; they can be combined and would be equal to $e_{d j} F_{y j}$.

For the example building, floor torques are computed in accordance with the 1987 Mexico Federal District code (Gomez and Garcia-Ranz 1988), which specifies $\alpha=1.5, \delta=1.0$, and $\beta=0.1$. The shears in the resisting elements are computed by static analyses of the building for the lateral forces $\mathbf{F}_{y}$ and floor torques $\tilde{\mathbf{F}}_{\theta}$ (Table 4). The shears in columns of frame $A$ due to lateral forces $\mathbf{F}_{y}$ (column 1, Table 4) and floor torques $\tilde{\mathbf{F}}_{\theta}$ (column 6 , Table 4) for analysis 1 are presented in column 2 of Table 5. Similar shears due to lateral forces $\mathbf{F}_{y}$ and floor torques $\tilde{\mathbf{F}}_{\theta}$ (column 8 , Table 4 ) for analysis 2 are presented in column 3 of Table 5 . The design shear, which is the larger of the shears obtained from the two analyses, is presented in column 4 of Table 5 .

\section{ANALYSIS WITHOUT USING CENTERS OF RIGIDITY}

The new approach to implement the code static-force procedure for asymmetric-plan building combines, according to a simple rule, the results of three sets of analyses. In each of these analyses, the forces are applied at the floor CMs, implying that the locations of the CRs are not needed. The three analyses are summarized in steps $1-3$, their superposition in step 4, and selection of the design value in step 5 .

1. With the code-specified lateral forces $F_{y j}$ applied at the floor CMs, analyze the building restricted to deform only in the $y$-direction [Fig. 6(a)]. This analysis can be implemented in standard computer programs for frame analysis by constraining the floor rotations. The resulting value of the desired response (force or deformation) is $r^{(1)}$.

2. With the code-specified lateral forces $F_{y j}$ applied at the floor CMs,

TABLE 4. Floor Torques for Example Building

\begin{tabular}{|c|c|c|c|c|c|c|c|}
\hline \multirow[b]{2}{*}{$\begin{array}{c}\text { Floor } \\
j \\
(1)\end{array}$} & \multirow[b]{2}{*}{$\begin{array}{c}F_{y i} \\
(\mathrm{kN}) \\
(2)\end{array}$} & \multirow[b]{2}{*}{$\begin{array}{c}e_{s j} \\
(\mathrm{~m}) \\
(3)\end{array}$} & \multirow[b]{2}{*}{$\begin{array}{c}b_{j} \\
(\mathrm{~m}) \\
(4)\end{array}$} & \multicolumn{2}{|c|}{ Analysis 1} & \multicolumn{2}{|c|}{ Analysis 2} \\
\hline & & & & $\begin{array}{c}e_{d j}=1.5 e_{s j} \\
+0.1 b \\
(\mathrm{~m}) \\
(5)\end{array}$ & $\begin{array}{c}\tilde{F}_{0 j}= \\
e_{d j} F_{y j} \\
(\mathrm{kN}-\mathrm{m}) \\
(6)\end{array}$ & $\begin{array}{c}e_{d j}=e_{s j}- \\
0.1 b \\
(\mathrm{~m}) \\
(7)\end{array}$ & $\begin{array}{c}\tilde{F}_{\theta j}=e_{d j} F_{y j} \\
(\mathrm{kN}-\mathrm{m}) \\
(8)\end{array}$ \\
\hline 4 & 5.54 & 0.99 & 10 & 2.485 & 13.77 & -0.010 & -0.05 \\
\hline 3 & 4.15 & 1.17 & 10 & 2.755 & 11.43 & 0.170 & 0.07 \\
\hline 2 & 5.54 & 7.55 & 20 & 13.325 & 73.82 & 5.55 & 30.75 \\
\hline 1 & 2.77 & -1.41 & 20 & -0.115 & -0.32 & -3.41 & -9.45 \\
\hline
\end{tabular}

TABLE 5. Shears in Columns of Frame A of Example Building

\begin{tabular}{c|c|c|c}
\hline $\begin{array}{c}\text { Floor } \\
j\end{array}$ & $\begin{array}{c}\text { Shear from Analysis 1 } \\
(\mathrm{kN}) \\
(1)\end{array}$ & $\begin{array}{c}\text { Shear from Analysis 2 } \\
(\mathrm{kN}) \\
(3)\end{array}$ & $\begin{array}{c}\text { Design shears } \\
(\mathrm{kN}) \\
(4)\end{array}$ \\
\hline 4 & 1.52 & 1.11 & 1.52 \\
3 & 2.67 & 1.91 & 2.67 \\
2 & 4.06 & 2.77 & 4.06 \\
1 & 4.43 & 2.85 & 4.43 \\
\hline
\end{tabular}




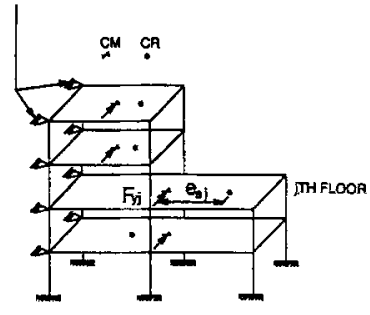

(a) Step 1

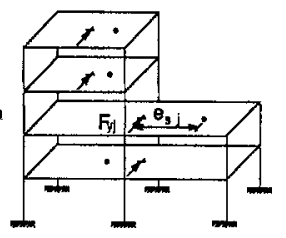

(b) Step 2

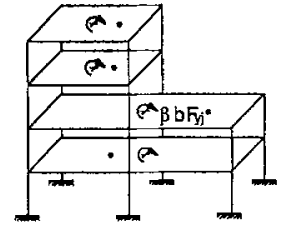

(c) Step 3

FIG. 6. Three Steps in Analysis by New Approach

TABLE 6. Shears in Columns of Frame A of Example Building by New Approach

\begin{tabular}{c|c|c|c|c|c|c}
\hline \hline $\begin{array}{c}\text { Floor } \\
j\end{array}$ & $\begin{array}{c}V_{j}^{(1)} \\
(2)\end{array}$ & $\begin{array}{c}V_{j}^{(2)} \\
(3)\end{array}$ & $\begin{array}{c}V_{j}^{(3)} \\
(4)\end{array}$ & $\begin{array}{c}V_{j}^{(\mathrm{a})}=-0.5 V_{j}^{(1)} \\
+1.5 V_{j}^{(2)}+V_{j}^{(3)} \\
(5)\end{array}$ & $\begin{array}{c}V_{j}^{(b)}=+V_{j}^{(2)} \\
-V_{j}^{(3)} \\
(6)\end{array}$ & $\begin{array}{c}\text { Design } \\
\text { shear } \\
(7)\end{array}$ \\
\hline 4 & 1.11 & 1.27 & 0.17 & 1.52 & 1.11 & 1.52 \\
3 & 1.91 & 2.21 & 0.31 & 2.67 & 1.91 & 2.67 \\
2 & 2.13 & 3.16 & 0.39 & 4.06 & 2.77 & 4.06 \\
1 & 2.40 & 3.39 & 0.54 & 4.43 & 2.85 & 4.43 \\
\hline \hline
\end{tabular}

analyze the asymmetric-plan building as a three-dimensional system to obtain the value $r^{(2)}$ of the desired response [Fig. $6(b)$ ].

3 . Analyze the asymmetric-plan system for the code-specified floor torques $=\beta b_{i} F_{y j}$ to obtain the value $r^{(3)}$ of the desired response [Fig. $\left.6(c)\right]$.

4. Obtain the responses $r^{(a)}$ and $r^{(b)}$ associated with design eccentricities of $(1 a)$ and $(1 b)$, respectively, by combining $r^{(1)}, r^{(2)}$, and $r^{(3)}$ as follows:

$r^{(a)}=(1-\alpha) r^{(1)}+\alpha r^{(2)}+r^{(3)}$
$r^{(b)}=(1-\delta) r^{(1)}+\delta r^{(2)}-r^{(3)}$

In each of (23) and (24), the algebraic sign of $r^{(3)}$ should be the one that increases the magnitude obtained from the sum of the first two terms.

5. The design value of the desired response is the larger of two values $r^{(a)}$ and $r^{(b)}$. If the design code includes a restriction that the design values should not be reduced because of torsion, the design value of the desired response is the largest of the three values $r^{(a)}, r^{(b)}$, and $r^{(1)}$.

For the example building, shears in the columns of frame A from the three analyses, summarized in steps 1,2 , and 3 , are presented in columns 2,3 , and 4 , respectively, of Table 6 . Combination of these three values in accordance with (23) and (24) provide $V^{(a)}$ and $V^{(b)}$ in columns 5 and 6 , respectively. The larger of $V^{(a)}$ and $V^{(b)}$ gives the design shears in column 7 of Table 6.

\section{EQUIVALENCE OF TWO APPROACHES}

The equivalence of the two approaches-the new approach and the procedure using CRs, which for convenience is called the standard approach- 
is demonstrated next by the principle of superposition; a more formal mathematical proof is included in Appendix I. As mentioned previously, the load condition applied in the standard approach [Fig. 5(a)] is equivalent to superposition of three load cases: (1) Lateral forces $\mathbf{F}_{y j}$ at the floor CRs [Fig. 5(b)]; (2) the floor torques $=\gamma e_{s j} F_{y j}$ [Fig. 5(c)]; and (3) the floor torques $=\beta b_{j} F_{y j}[$ Fig. $5(d)]$.

Step 1 of the new approach [Fig. 6(a)] is equivalent to the first load case of the standard approach [Fig. 5(b)] because the lateral stiffness matrix $\mathbf{K}_{y y}$ and the lateral forces $\mathbf{F}_{y}$ are the same in the two cases, and floor rotations are absent in both cases: they have been prevented in the new approach and they do not occur in the standard approach because the forces are applied at the CRs. Step 3 of the new approach [Fig. 6(c)] is equivalent to the third load case of the standard approach [Fig. $5(d)$ ] because in both cases the forces applied are the same pure floor torques.

The second load case of the standard approach includes floor torques $=$ $\gamma e_{s j} F_{y j}$ [Figs. 5(c) and 7(a)]; which can be expressed as superposition of three load cases: (1) Lateral forces $=\gamma \mathbf{F}_{y j}$ at the CRs [Fig. 7(b)]; (2) floor torques $=\gamma e_{s j} F_{y j}[$ Fig. $7(c)]$; and (3) lateral forces $=-\gamma \mathbf{F}_{y j}[$ Fig. $7(d)]$ at the CRs. The first two load cases combined are equivalent to the application of the lateral forces $=\gamma \mathbf{F}_{y j}$ at the CMs [Fig. 7(e)]. Consequently, the load case of Fig. 5(c) or Fig. 7(a) is equivalent to $\gamma$ times the results of step 2 of the new approach [Fig. $6(b)$ ] minus $\gamma$ times the results of step 1 of the new approach [Fig. 6(a)].

Restating the conclusions of the preceding two paragraphs, the response determined by the standard approach $r$ is obtained as

$r=r^{(1)}+\gamma\left(r^{(2)}-r^{(1)}\right) \pm r^{(3)}$

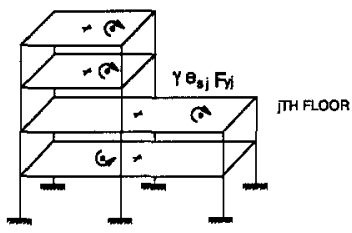

(a)

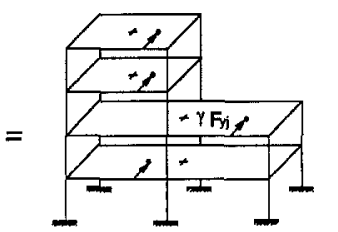

(b)

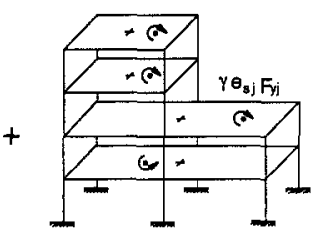

(c)

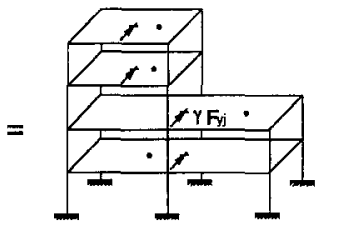

(e)

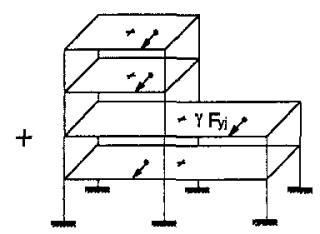

(d)

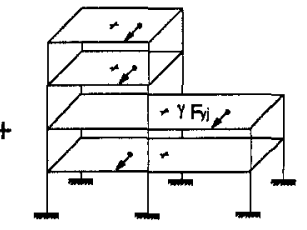

$($ )

FIG. 7. Second Load Case of Approach Using Centers of Rigidity as Combination of Steps 1 and 2 of New Approach 
$r=(1-\gamma) r^{(1)}+\gamma r^{(2)} \pm r^{(3)}$

which is a combined version of (23) and (24).

For the example building, shears in the columns of frame A computed from the two approaches are the same as demonstrated by the identical results obtained in columns 5-7 of Table 6 and columns $2-4$ of Table 5 .

The computational effort required to implement the analyses in steps 2 and 3 of the new approach is the same as that in the standard approach to implement the analyses for the two values of the design eccentricity. The computational effort required in the step 1 of the new approach is less than that required to determine the locations of the CRs in the standard approach beause of the supplemental analysis required in this approach. Therefore, total computational efforts required in the former approach would be slightly less than that required in the latter.

\section{CONCLUSIONS}

A new approach is presented for code lateral force analysis of asymmetricplan buildings without locating the centers of rigidity, an often confusing and cumbersome process. This procedure combines the results from three static analyses that can be implemented directly on most commercially available computer programs for analysis of multistory buildings. The work presented in this paper should dispel the long-held view that locations of the centers of rigidity must be determined to implement the code procedure, thereby removing one of the major difficulties in building analysis.

\section{ACKNOWLEDGMENTS}

This research investigation is supported by the National Science Foundation under grant BCS-8921932. The writers are grateful for this support. The writers are also thankful to the professors J. L. Humar, E. Rosenblueth, and W. K. Tso for their comments on the initial draft of this paper.

\section{APPENDIX I. MATHEMATICAL PROOF OF EQUIVALENCE OF TWO APPROACHES}

The equivalence of the two approaches, outlined in this paper, is established in this appendix for buildings with orthogonal lateral resisting elements and rigid diaphragm. This is achieved by demonstrating that the deformations at the floor CRs of the building obtained by the two approaches are identical. For rigid-diaphragm buildings, deformations at all other points on the floor plan as well as forces in resisting elements would obviously be identical from the two approaches.

\section{Equations of Equilibrium}

The equation of equilibrium with respect to the CMs are

$$
\left[\begin{array}{ll}
\mathbf{K}_{y y} & \mathbf{K}_{y \theta} \\
\mathbf{K}_{\theta y} & \mathbf{K}_{\boldsymbol{\theta} \theta}
\end{array}\right]\left\{\begin{array}{l}
\mathbf{u}_{y} \\
\mathbf{u}_{\theta}
\end{array}\right\}=\left\{\begin{array}{l}
\mathbf{F}_{y} \\
\mathbf{F}_{\theta}
\end{array}\right\}
$$

in which $\mathbf{u}_{y}$ and $\mathbf{u}_{\theta}=$ vectors of lateral and torsional displacements, respectively, at the CMs; and $\mathbf{F}_{y}$ and $\mathbf{F}_{\theta}=$ vectors of lateral forces and floor 
torques, respectively, applied at the CMs. Similarly, equation of equilibrium with respect to the CRs is

$$
\left[\begin{array}{ll}
\tilde{\mathbf{K}}_{y y} & \tilde{\mathbf{K}}_{y \theta} \\
\tilde{\mathbf{K}}_{\theta y} & \tilde{\mathbf{K}}_{\theta \theta}
\end{array}\right]\left\{\begin{array}{l}
\tilde{\mathbf{u}}_{y} \\
\tilde{\mathbf{u}}_{\theta}
\end{array}\right\}=\left\{\begin{array}{l}
\tilde{\mathbf{F}}_{y} \\
\tilde{\mathbf{F}}_{\theta}
\end{array}\right\}
$$

in which $\tilde{\mathbf{u}}_{y}$ and $\tilde{\mathbf{u}}_{\theta}=$ vectors of lateral and torsional displacements, respectively, at the CRs; and $\tilde{\mathbf{F}}_{y}$ and $\tilde{\mathbf{F}}_{\theta}=$ vectors of lateral forces and floor torques, respectively, applied at the CRs.

For buildings with rigid diaphragms and orthogonal arrangement of the lateral-load-resisting elements, various submatrices in the stiffness matrix of $(28)$ are related to those of $(27)$

$$
\begin{aligned}
& \tilde{\mathbf{K}}_{y y}=\mathbf{K}_{y y} \\
& \tilde{\mathbf{K}}_{y \theta}=\mathbf{K}_{y \theta}-\mathbf{K}_{y y} \mathbf{e}_{s} \\
& \tilde{\mathbf{K}}_{\theta \mathbf{y}}=\mathbf{K}_{\boldsymbol{\theta} y}-\mathbf{e}_{s} \mathbf{K}_{y y} \\
& \check{\mathbf{K}}_{\theta \theta}=\mathbf{K}_{\theta \theta}+\mathbf{e}_{s} \mathbf{K}_{y y} \mathbf{e}_{s}-\mathbf{e}_{s} \mathbf{K}_{y \theta}-\mathbf{K}_{\theta y} \mathbf{e}_{s}
\end{aligned}
$$

in which $\mathbf{e}_{s}=$ diagonal matrix with diagonal terms $=\boldsymbol{e}_{s j}$.
From the definition of the center of rigidity, if only $\tilde{\mathbf{F}}_{y}\left(=\mathbf{F}_{y}\right)$ is applied at the CRs, i.e. $\tilde{\mathbf{F}}_{\theta}=0$, then the system would undergo pure translation with $\tilde{\mathbf{u}}_{\theta}=0$. Thus, (28) becomes

$$
\begin{aligned}
& \tilde{\mathbf{K}}_{y y} \tilde{\mathbf{u}}_{y}=\mathbf{F}_{y} \\
& \tilde{\mathbf{K}}_{\theta y} \tilde{\mathbf{u}}_{y}=0
\end{aligned}
$$

Eq. (30a) is solved for $\tilde{\mathbf{u}}_{y}$

$$
\tilde{\mathbf{u}}_{y}=\tilde{\mathbf{K}}_{y y}^{-1} \mathbf{F}_{y}
$$

and (31) is substituted in $(30 b)$ to obtain

$\tilde{\mathbf{K}}_{\theta y} \tilde{\mathbf{K}}_{y y}^{-1} \mathbf{F}_{y}=0$

Utilizing (29a) and (29c), (32) becomes

$$
\left(\mathbf{K}_{\theta \theta}-\mathbf{e}_{s} \mathbf{K}_{y y}\right)\left(\mathbf{K}_{y y}^{-1}\right) \mathbf{F}_{y}=\mathbf{0}
$$

which is further simplified to give

$$
\mathbf{K}_{\theta y} \tilde{\mathbf{K}}_{y y}^{-1} \mathbf{F}_{y}=\mathbf{e}_{y} \mathbf{F}_{y}
$$

Eqs. (32) and (34) would be utilized in subsequent sections to demonstrate the equivalene of the two approaches.

\section{Deformation at CRs by Standard Approach}

As mentioned previously, the load condition applied in this approach is equivalent to applying the lateral forces $=F_{y j}$ at the CRs and floor torques $=-\left(\gamma e_{s j} F_{y j} \pm \beta b_{j} F_{y j}\right)$; the negative sign for the floor torques is used to be compatible with the direction of the rotational degrees of freedom considered positive in the counterclockwise direction (Fig. 1). as

The deformations $\tilde{\mathbf{u}}_{y}$ and $\tilde{\mathbf{u}}_{\theta}$ at the CRs are computed by solving the (28) 
$\left[\begin{array}{ll}\tilde{\mathbf{K}}_{y y} & \tilde{\mathbf{K}}_{y \theta} \\ \tilde{\mathbf{K}}_{\theta y} & \tilde{\mathbf{K}}_{\theta \theta}\end{array}\right]\left\{\begin{array}{l}\tilde{\mathbf{u}}_{y} \\ \tilde{\mathbf{u}}_{\theta}\end{array}\right\}=\left\{\begin{array}{l}\mathbf{F}_{y} \\ -\left(\gamma \mathbf{e}_{s} \mathbf{F}_{y}+\beta \mathbf{b F} \mathbf{F}_{y}\right)\end{array}\right\}$

in which $\mathbf{b}=$ diagonal matrix with diagonal elements $=b_{j}$.

Solving the first equation of (35) for $\tilde{\mathbf{u}}_{y}$ in terms of $\tilde{\mathbf{u}}_{\theta}$ gives

$\tilde{\mathbf{u}}_{y}=\tilde{\mathbf{K}}_{y y}^{-1} \mathbf{F}_{y}-\tilde{\mathbf{K}}_{y y}^{-1} \tilde{\mathbf{K}}_{y \theta} \tilde{\mathbf{u}}_{\boldsymbol{\theta}}$

Utilizing (36), second equation of (35) becomes

$\tilde{\mathbf{K}}_{\theta y} \tilde{\mathbf{K}}_{y y}^{-1} \mathbf{F}_{y}-\tilde{\mathbf{K}}_{\theta y} \tilde{\mathbf{K}}_{y y}^{-1} \tilde{\mathbf{K}}_{y \theta} \tilde{\mathbf{u}}_{\theta}+\tilde{\mathbf{K}}_{\theta \theta} \tilde{\mathbf{u}}_{\theta}=-\left(\gamma \mathbf{e}_{s} \mathbf{F}_{y}+\beta \mathbf{b} \mathbf{F}_{y}\right)$

which gives

$\tilde{\mathbf{u}}_{\theta}=-\left(\overline{\mathbf{K}}_{\theta \theta}-\tilde{\mathbf{K}}_{\theta y} \tilde{\mathbf{K}}_{y y}^{-1} \tilde{\mathbf{K}}_{y \theta}\right)^{-1}\left(\gamma \mathbf{e}_{s} \mathbf{F}_{y}+\beta \mathbf{b} \mathbf{F}_{y}+\tilde{\mathbf{K}}_{\theta y} \tilde{\mathbf{K}}_{y y}^{-1} \mathbf{F}_{y}\right)$

To demonstrate the equivalence of the two approaches, it is useful to write the submatrices $\tilde{\mathbf{K}}_{y y}, \tilde{\mathbf{K}}_{y \theta}, \tilde{\mathbf{K}}_{\theta y}$, and $\tilde{\mathbf{K}}_{\theta \theta}$ defined at the CR in terms of the submatrices $\mathbf{K}_{y y}, \mathbf{K}_{y \theta}, \mathbf{k}_{\theta y}$, and $\mathbf{K}_{\theta \theta}$ defined at the CM. This is achieved by utilizing (29) to obtain

$\left(\tilde{\mathbf{K}}_{\theta \theta}-\tilde{\mathbf{K}}_{\theta y} \tilde{\mathbf{K}}_{y y}^{-1} \tilde{\mathbf{K}}_{y \theta}\right)=\mathbf{K}_{\theta \theta}+\mathbf{e}_{s} \mathbf{K}_{y y} \mathbf{e}_{s}-\mathbf{e}_{s} \mathbf{K}_{y \theta}-\mathbf{K}_{\theta y} \mathbf{e}_{s}$

$-\left(\mathbf{K}_{\theta y}-\mathbf{e}_{s} \mathbf{K}_{y y}\right) \mathbf{K}_{y y}^{-1}\left(\mathbf{K}_{y \theta}-\mathbf{K}_{y y} \mathbf{e}_{s}\right)=\mathbf{K}_{\theta \theta}+\mathbf{e}_{s} \mathbf{K}_{y y} \mathbf{e}_{s}-\mathbf{e}_{s} \mathbf{K}_{y \theta}-\mathbf{K}_{\theta y} \mathbf{e}_{s}$

$-\mathbf{K}_{\theta y} \tilde{\mathbf{K}}_{y y}^{-1} \mathbf{K}_{y \theta}+\mathbf{K}_{\theta y} \mathbf{K}_{y y}^{-1} \mathbf{K}_{y y} \mathbf{e}_{s}+\mathbf{e}_{s} \mathbf{K}_{y \theta}-\mathbf{e}_{s} \mathbf{K}_{y y} \mathbf{e}_{s}=\left(\mathbf{K}_{\theta \theta}-\mathbf{K}_{\theta y} \tilde{\mathbf{K}}_{y y}^{-1} \mathbf{K}_{y \theta}\right)$

and

$\tilde{\mathbf{K}}_{y y}^{-1} \overline{\mathbf{K}}_{y \boldsymbol{\theta}}=\mathbf{K}_{y y}^{-1}\left(\mathbf{K}_{y \theta}-\mathbf{K}_{y y} \mathbf{e}_{s}\right)=\left(\mathbf{K}_{y y}^{-1} \mathbf{K}_{y \theta}-\mathbf{e}_{s}\right)$

Utilizing (32), (39), and (40) in (38) gives

$\tilde{\mathbf{u}}_{\theta}=-\left(\mathbf{K}_{\theta \theta}-\mathbf{K}_{\theta y} \mathbf{K}_{y y}^{-1} \mathbf{K}_{y \theta}\right)^{-1} \gamma \mathbf{e}_{s} \mathbf{F}_{y}-\left(\mathbf{K}_{\theta \theta}-\mathbf{K}_{\theta y} \mathbf{K}_{y y}^{-1} \mathbf{K}_{y \theta}\right)^{-1} \beta \mathbf{b F} \mathbf{F}_{y}$.

Replacing $\tilde{\mathbf{u}}_{\theta}$ obtained from (41) into (36) give

$\tilde{\mathbf{u}}_{y}=\mathbf{K}_{y y}^{-1} \mathbf{F}_{y}+\mathbf{K}_{y y}^{-1} \mathbf{K}_{y \theta}\left(\mathbf{K}_{\theta \theta}-\mathbf{K}_{\theta y} \mathbf{K}_{y y}^{-1} \mathbf{K}_{y \theta}\right)^{-1} \gamma \mathbf{e}_{s} \mathbf{F}_{y}+\mathbf{K}_{y y}^{-1} \mathbf{K}_{y \theta}\left(\mathbf{K}_{\theta \theta}\right.$

$\left.-\mathbf{K}_{\theta y} \mathbf{K}_{y y}^{-1} \mathbf{K}_{y \theta}\right)^{-1} \beta \mathbf{b F} \mathbf{F}_{y}-\mathbf{e}_{s}\left(\mathbf{K}_{\theta \theta}-\mathbf{K}_{\theta y} \mathbf{K}_{y y}^{-1} \mathbf{K}_{y \theta}\right)^{-1} \gamma \mathbf{e}_{s} \mathbf{F}_{\boldsymbol{y}}-\mathbf{e}_{s}\left(\mathbf{K}_{\theta \theta}\right.$

$\left.-\mathbf{K}_{\theta y} \mathbf{K}_{y y}^{-1} \mathbf{K}_{y \boldsymbol{\theta}}\right)^{-1} \beta \mathbf{b F} \mathbf{F}_{y}$

\section{Deformation at CRs by New Approach}

The deformations at the CMs and CRs from step 1 would be identical and would be equal to

$\tilde{\mathbf{u}}_{y}^{(1)}=\tilde{\mathbf{u}}_{y}^{(1)}=\tilde{\mathbf{K}}_{y y}^{-1} \mathbf{F}_{y}=\mathbf{K}_{y y}^{-1} \mathbf{F}_{y}$

since the torsional deformations are

$\tilde{\mathbf{u}}_{\theta}^{(1)}=0$

Deformations at the CMs in step 2 are computed from

$\left[\begin{array}{ll}\mathbf{K}_{y y} & \mathbf{K}_{y \theta} \\ \mathbf{K}_{\theta y} & \mathbf{K}_{0 \theta}\end{array}\right]\left\{\begin{array}{l}\mathbf{u}_{y}^{(2)} \\ \mathbf{u}_{\theta}^{(2)}\end{array}\right\}=\left\{\begin{array}{c}\mathbf{F}_{y} \\ 0\end{array}\right\}$

which leads to 
$\mathbf{u}_{y}^{(2)}=\mathbf{K}_{y y}^{-1} \mathbf{F}_{y}-\mathbf{K}_{y y}^{-1} \mathbf{K}_{y \theta} \mathbf{u}_{\theta}^{(2)}$

and

$\mathbf{u}_{\theta}^{(2)}=-\left(\mathbf{K}_{\theta \theta}-\mathbf{K}_{\theta y} \mathbf{K}_{y y}^{-1} \mathbf{K}_{y \theta}\right)^{-1} \mathbf{K}_{\theta y} \mathbf{K}_{y y}^{-1} \mathbf{F}_{y}$

Utilizing (34) in (47) gives

$\mathbf{u}_{\theta}^{(2)}=-\left(\mathbf{K}_{\theta \theta}-\mathbf{K}_{\theta y} \mathbf{K}_{y y}^{-1} \mathbf{K}_{y \theta}\right)^{-1} \mathbf{e}_{s} \mathbf{F}_{y}$

Replacing (48) in (46) gives

$\mathbf{u}_{y}^{(2)}=\mathbf{K}_{y y}^{-1} \mathbf{F}_{y}+\mathbf{K}_{y y}^{-1} \mathbf{K}_{y \theta}+\left(\mathbf{K}_{\theta \theta}-\mathbf{K}_{\theta y} \mathbf{K}_{y y}^{-1} \mathbf{K}_{y \theta}\right)^{-1} \mathbf{e}_{s} \mathbf{F}_{y}$

Having determined the deformations at the CMs, deformations at the CRs are obtained as

$\tilde{\mathbf{u}}_{y}^{(2)}=\mathbf{u}_{y}^{(2)}+\mathbf{e}_{s} \mathbf{u}_{\theta}^{(2)}$

and

$\tilde{\mathbf{u}}_{\theta}^{(2)}=\mathbf{u}_{\theta}^{(2)}$

Utilizing (48) and (49) in (50) and (51) leads to

$\tilde{\mathbf{u}}_{y}^{(2)}=\mathbf{K}_{y y}^{-1} \mathbf{F}_{y}+\mathbf{K}_{y y}^{-1} \mathbf{K}_{y \theta}\left(\mathbf{K}_{\theta \theta}-\mathbf{K}_{\theta y} \mathbf{K}_{y y}^{-1} \mathbf{K}_{y \theta}\right)^{-1} \mathbf{e}_{s} \mathbf{F}_{y}$

$-\mathbf{e}_{s}\left(\mathbf{K}_{\theta \theta}-\mathbf{K}_{\theta y} \mathbf{K}_{y y}^{-1} \mathbf{K}_{y \theta}\right)^{-1} \mathbf{e}_{s} \mathbf{F}_{y}$

$\tilde{\mathbf{u}}_{\theta}^{(2)}=-\left(\mathbf{K}_{\theta \theta}-\mathbf{K}_{\theta y} \mathbf{K}_{y y}^{-1} \mathbf{K}_{y \theta}\right)^{-1} \mathbf{e}_{s} \mathbf{F}_{y}$

Similarly, deformations in step 3 due to floor torques $=-\beta \mathbf{b} \mathbf{F}_{y}$ are computed from

$\left[\begin{array}{ll}\mathbf{K}_{y y} & \mathbf{K}_{y \theta} \\ \mathbf{K}_{\theta y} & \mathbf{K}_{\theta \theta}\end{array}\right]\left\{\begin{array}{l}\mathbf{u}_{y}^{(3)} \\ \mathbf{u}_{\theta}^{(3)}\end{array}\right\}=\left\{\begin{array}{c}0 \\ -\mathbf{\beta b F}_{y}\end{array}\right\}$

and are given by

$\mathbf{u}_{\theta}^{(3)}=-\left(\mathbf{K}_{\theta \theta}-\mathbf{K}_{\theta y} \mathbf{K}_{y y}^{-1} \mathbf{K}_{y \theta}\right)^{-1} \beta \mathbf{b F} \mathbf{F}_{y}$

$\mathbf{u}_{y}^{(3)}=\mathbf{K}_{y y}^{-1} \mathbf{K}_{y \theta}\left(\mathbf{K}_{\theta \theta}-\mathbf{K}_{\theta y} \mathbf{K}_{y y}^{-1} \mathbf{K}_{y \theta}\right)^{-1} \beta \mathbf{b F} \mathbf{F}_{y}$

And the deformations at the CRs are given by

$\tilde{\mathbf{u}}_{y}^{(3)}=\mathbf{K}_{y y}^{-1} \mathbf{K}_{y \theta}\left(\mathbf{K}_{\theta \theta}-\mathbf{K}_{\theta y} \mathbf{K}_{y y}^{-1} \mathbf{K}_{y \theta}\right)^{-1} \boldsymbol{\beta} \mathbf{b F} \mathbf{F}_{y}$

$-\mathbf{e}_{s}\left(\mathbf{K}_{\theta \theta}-\mathbf{K}_{\theta y} \mathbf{K}_{y y}^{-1} \mathbf{K}_{y \theta}\right)^{-1} \beta \mathbf{b} \mathbf{F}_{y}$

and

$\overline{\mathbf{u}}_{\theta}^{(3)}=-\left(\mathbf{K}_{\theta \theta}-\mathbf{K}_{\theta y} \mathbf{K}_{y y}^{-1} \mathbf{K}_{y \theta}\right)^{-1} \beta \mathbf{b F} \mathbf{F}_{y}$

Combining the deformations obtained in steps 1,2 , and 3 according to the combination rule of (26) gives

$$
\begin{aligned}
& \tilde{\mathbf{u}}_{y}=\tilde{\mathbf{u}}_{y}^{(1)}+\gamma\left(\tilde{\mathbf{u}}_{y}^{(2)}-\tilde{\mathbf{u}}_{y}^{(1)}\right)+\tilde{\mathbf{u}}_{y}^{(3)}=\mathbf{K}_{y y}^{-1} \mathbf{F}_{y}+\mathbf{K}_{y y}^{-1} \mathbf{K}_{y \theta}\left(\mathbf{K}_{\theta \theta}\right. \\
& \left.-\mathbf{K}_{\theta y} \mathbf{K}_{y y}^{-1} \mathbf{K}_{y \theta}\right)^{-1} \gamma \mathbf{e}_{s} \mathbf{F}_{y}+\mathbf{K}_{y y}^{-1} \mathbf{K}_{y \theta}\left(\mathbf{K}_{\theta \theta}-\mathbf{K}_{\theta y} \mathbf{K}_{y y}^{-1} \mathbf{K}_{y \theta}\right)^{-1} \beta \mathbf{b} \mathbf{F}_{y} \\
& -\mathbf{e}_{s}\left(\mathbf{K}_{\theta \theta}-\mathbf{K}_{\theta y} \mathbf{K}_{y y}^{-1} \mathbf{K}_{y \theta}\right)^{-1} \gamma \mathbf{e}_{s} \mathbf{F}_{y}-\mathbf{e}_{s}\left(\mathbf{K}_{\theta \theta}-\mathbf{K}_{\theta y} \mathbf{K}_{y y}^{-1} \mathbf{K}_{y \theta}\right)^{-1} \beta \mathbf{b} \mathbf{F}_{y}
\end{aligned}
$$

and 


$$
\begin{aligned}
& \tilde{\mathbf{u}}_{\theta}=\tilde{\mathbf{u}}_{\theta}^{(1)}+\gamma\left(\tilde{\mathbf{u}}_{\theta}^{(2)}-\tilde{\mathbf{u}}_{\theta}^{(1)}\right)+\tilde{\mathbf{u}}_{\theta}^{(3)}=-\left(\mathbf{K}_{\theta \theta}-\mathbf{K}_{\theta y} \mathbf{K}_{y y}^{-1} \mathbf{K}_{y \theta}\right)^{-1} \gamma \mathbf{e}_{s} \mathbf{F}_{y} \\
& \text { - }\left(\mathbf{K}_{\theta \theta}-\mathbf{K}_{\theta y} \mathbf{K}_{y y}^{-1} \mathbf{K}_{y \theta}\right)^{-1} \beta \mathbf{b} \mathbf{F}_{y}
\end{aligned}
$$

Eqs. (59) and (60) are identical to (42) and (41), respectively, indicating that the response obtained by the new approach is identical to that obtained by the standard approach.

\section{APPENDIX II. REFERENCES}

Cheung, V. W.-T., and Tso, W. K. (1986). "Eccentricity in irregular multistory buildings." Can. I. Civ. Engrg., 13(1), 46-52.

Earthquake resistant regulations, a world list. (1992). Int. Assoc. for Earthquake Engrg., Tokyo, Japan.

Gomez, R., and Garcia-Ranz, F. (1988). "The Mexico earthquake of September 19, 1985-complementary technical norms for earthquake resistant design." Earthquake Spectra, 4(3), 441-460.

Hejal, R., and Chopra, A. K. (1987). "Earthquake response of torsionally-coupled buildings." Report no. UCB/EERC-87/20, Earthquake Engrg. Res. Ctr., Univ. of California, Berkeley, Calif.

Humar, J. L. (1984). "Design for seismic torsional forces." Can. J. Civ. Engrg., 12(2), 150-163.

National building code of Canada. (1990). Assoc. Committee on the Nat. Build. Code, Nat. Res. Council of Canada, Ottawa, Ontario.

New Zealand standard NZS 4203: code of practice for general structural design loadings for buildings. (1984). Standards Assoc. of New Zealand, Willington, New Zealand.

Poole, R. A. (1977). "Analysis for torsion employing provisions of NZRS 4203: 1974." Bull. of the New Zealand Soc. for Earthquake Engrg., 10(4), 219-225.

Riddell, R., and Vasquez, J. (1984). "Existence of centers of resistance and torsional uncoupling of earthquake response of buildings." Proc., 8th World Conf. on Earthquake Engrg., 4, 187-194.

"Tentative provisions for the development of seismic regulations for buildings." (1978). ATC3-06, Appl. Tech. Council, Palo Alto, Calif.

Tso, W. K. (1990). "Static eccentricity concept for torsional moment estimations." J. Struct. Engrg., ASCE, 116(5), 1199-1212.

Tso, W. K., and Cheung, V. W.-T. (1986). "Decoupling of equations of equilibrium in lateral load analysis of multistory buildings." Comp. and Struct., 23(5), 679684.

Uniform building code. (1991). Int. Conf. of Build. Officials, Whittier, Calif.

Wilson, E. L., and Habibullah, A. (1984). SAP80 structural analysis programs for the static and dynamic finite element analysis of structures. Computers and Structures Inc., Berkeley, Calif. 